\title{
Articulación de procesos, flujos de información y conocimiento bajo criterios de infoconocimiento y sostenibilidad en el reporte corporativo
}

\author{
Process Articulation, Information Flow and Knowledge Flow under Info-Knowledge and Sustainability \\ Criteria in the Corporate Reports \\ Articulação de processos, fluxos de informação e conhecimento sob critérios de infoconhecimento e \\ sustentabilidade no relatório corporativo
}

Marisleidy Alba

Universidad Externado de Colombia, Colombia

marisleidy.alba@uexternado.edu.co

ORCID: http://orcid.org/0000-0001-6935-6097

DOI: https://doi.org/10.11144/Javeriana.cc19-47.apfi Redalyc: http://www.redalyc.org/articulo.oa? id $=151556994006$

César Beltrán Torres

Universidad Externado de Colombia, Colombia

ORCID: http://orcid.org/0000-0003-4635-1298

Fecha de recepción: 20 Septiembre 2017

Fecha de aprobación: 23 Abril 2018

\section{Resumen:}

La crisis ambiental, social y de credibilidad demanda a las organizaciones reportes corporativos que den cuenta de sus prácticas empresariales, de sostenibilidad y transparencia; este artículo busca identificar las condiciones necesarias y las potencialidades que trae la articulación del reporte integrado (RI) propuesto por el Internacional Integrated Reporting Council (IIRC) y el modelo de Gestión de infoconocimiento para cadenas de suministro (Giccs). El desarrollo de la investigación se basa en un análisis cualitativo conceptual, por medio de una comparación de las categorías que fundamentan el modelo Giccs y el RI, validada con una revisión y clasificación de la literatura. Dentro de los resultados más relevantes están el identificar que el RI se construye por medio de información, por tal razón, requiere una gestión de los flujos de información y de conocimiento de las áreas y procesos de la organización; al involucrar el Giccs se garantiza la integración de los flujos de información y de conocimiento. Otro hallazgo fue ubicar al RI como un aliado fundamental en la identificación de los flujos de información y conocimiento que deben ser gestionados, de tal suerte que por medio del modelo Giccs se pueda definir una estructura clara para estos flujos, y así también se permitan integrar elementos para aportar a los procesos organizacionales y finalmente su integración a escalas superiores. El modelo de gestión del infoconocimiento Giccs y el RI se cohesionan para garantizar en el proceso de confección del reporte la estandarización de las entradas, el registro de información, conservación y circulación de la información y el conocimiento, contribuyendo con una mejor relevación organizacional y con ello a la transparencia y a la confianza en la sociedad.

Palabras clave: generación de valor, gestión de la información, gestión del conocimiento, reportes corporativos, sostenibilidad, transparencia.

\begin{abstract}
:
The environmental, social and credibility crisis requires the organizations to do corporate reports accounting for their managerial, sustainability and transparence practices. This article seeks to identify the necessary conditions as well as the affordances offered by the articulation of the integrated report (IR) proposed by the International Integrated Reporting Council (IIRC) and the model of Info-Knowledge Management for Supply Chains (IKMSC). This research is developed based on a conceptual qualitative analysis, carrying out a comparison between the categories providing the grounds for the IKMSC model and the IR, validated through a review and classification of the literature. The most relevant results include to identify that the IR is built out of information. Therefore, it requires to carry out an information and knowledge flow management throughout the organization areas and processes; putting the IKMSC into practice will ensure the integration of information flows and knowledge flows. Additionally, it was found that the IR serves as a fundamental ally in the identification of the information and knowledge flows that should be arranged, which will allow the IKMSC model to define a clear structure for these flows. It will also allow to integrate elements contributing to the organizational processes and, finally, to integrate them at a higher extent. The IKMSC model and the IR draw together to guarantee the standardization of entries, the recording of information, the preservation and circulation of the information and knowledge throughout the report preparation process, which contribute to a better organizational disclosure and thus allowing the transparence and confidence among the society. Keywords: value generation, information management, knowledge management, corporate reports, sustainability, transparence.
\end{abstract}




\section{Resumo:}

A crise ambiental, social e de credibilidade exige que as organizações relatórios corporativos informem suas práticas empresariais, de sustentabilidade e transparência. Este artigo visa identificar as condições necessárias e as potencialidades que traz a articulação do relatório integrado (RI) proposto pelo Internacional Integrated Reporting Council (IIRC) e o modelo de Gestão de infoconhecimento para cadeias de fornecimento (Giccs). O desenvolvimento da pesquisa baseia-se em uma análise qualitativo conceitual, através de um comparativo das categorias que fundamentam o modelo Giccs e o RI, validada com uma revisão e classificação da literatura. Dentre os resultados mais relevantes estão o identificar que o RI é construído por meio de informação, por tal razão, requer uma gestão dos fluxos de informação e conhecimento das áreas e processos da organização; ao involucrar o Giccs a integração dos fluxos de informação e conhecimento é garantida. Outro achado foi localizar o RI como aliado-chave na identificação dos fluxos de informação e conhecimento a ser geridos, de tal sorte que por meio do modelo Giccs pudera-se definir uma estrutura clara para esses fluxos, e mesmo assim permitem integrar elementos para aportar aos processos organizacionais e finalmente sua integração a escalas superiores. O modelo de gestão do infoconhecimento Giccs e o RI são consorciados para garantir no processo de elaboração do relatório a padronização das entradas, o registro, conservação e circulação da informação e o conhecimento, contribuindo com uma melhor revelação organizacional e com isso à transparência e a confiança na sociedade.

Palavras-chave: geração de valor, gestão da informação, gestão do conhecimento, relatórios corporativos, sustentabilidade, transparência.

\section{Introducción}

La contabilidad ha evolucionado a lo largo de los diferentes sistemas de organización social de la producción y en cada uno de ellos se le ha permitido un mayor o menor nivel de fundamentación, sin importar cuál sea el sistema; tanto sus funciones como su fundamentación han estado relacionadas con la información, el control y la medición (Ijiri, 1976; Sunder, 2005). De las funciones históricas de la contabilidad referidas a la medición, control e información, se hará énfasis en las últimas dos, considerando el tipo de problema que se aborda. Así mismo, se tratará la contabilidad en el escenario global actual, enfocado no en los elementos de reconocimiento, medición o valoración contable, sino en la revelación de la información contable que las organizaciones entregan a la sociedad, es decir, la información del desempeño de dichas organizaciones.

El entorno global en el que operan las organizaciones impone retos de competitividad, rentabilidad, eficiencia y calidad, entre otros. Sin embargo, los retos más importantes han surgido de las profundas y crecientes crisis sociales y ambientales por las que atraviesa la humanidad, y en las que las organizaciones juegan un rol protagónico. La respuesta de las organizaciones ha sido involucrarse en diferentes programas de Responsabilidad Social Corporativa (RSC), los cuales involucran actividades como repensar los procesos productivos, la logística de distribución y abastecimiento, y un cambio en la revelación de la información sobre la forma en que la organización se han desempeñado con la sociedad y el medio ambiente. Sumado a la necesidad de revelación sobre RSC, es necesario mencionar que los diferentes escándalos financieros de los últimos años han generado un ambiente de desconfianza y pérdida de credibilidad en la información revelada por las organizaciones; esta situación obliga a pensar en nuevas formas de socialización de la información y en un papel cada vez más transparente por parte de los preparadores y aseguradores de la información contable.

Los grupos de interés (stakeholders) ya no se limitan a los inversores y reguladores, ahora la sociedad civil, los proveedores, el medio ambiente, las comunidades locales y los trabajadores, entre otros, están cada vez más interesados y formados en saber cómo se desempeñan las organizaciones, cuál es su aporte al cuidado del medio ambiente, a la generación de capacidades culturales de las comunidades cercanas, cómo se articulan en su cadena de suministro y cómo aportan en general a la creación de confianza y transparencia.

La información voluntaria que las organizaciones revelan ha venido creciendo de forma acelerada a nivel mundial, los emisores de información financiera se han preocupado porque sus estándares permitan revelar aspectos más allá de los tradicionales financieros y centrados en el corto plazo (Hahn y Kuhnen, 2013; KPMG, 2017; Toppinen, Li, Tuppura y Xiong, 2012; García-Benau, Sierra-García y Zorio-Grima, 2012). Como respuesta, han surgido diversas propuestas para involucrar información no financiera, prácticas de accountability y gestión ambiental que se traducen en nuevas prácticas organizacionales y en modelos de 
reporte y aseguramiento organizacional, por nombrar algunas: Global Reporting Initiative (GRI), Ethos, Pacto Global, los principios de Accountability AA1000, ISO 26000, The International <IR $>$ Framework (Integrated Reporting) del International Integrated Reporting Council (IIRC), Sustainability Accounting Standards (SASB), Enhanced Business Report (EBR) y The Connected Reporting Framework (CRF).

Las propuestas de reportes corporativos de RSC han tenido muy buena recepción en las diferentes organizaciones a nivel mundial, en gran medida como lo explican Bruhn y Zimmermann (2017), esto obedece a que la alta dirección las asume como un concepto estratégico y, por tanto, les permite promover la competitividad de una corporación, adoptando el principio de legitimidad social, y porque mejora su reputación e imagen de marca y sus relaciones con las partes interesadas y con el pblico en general.

La propuesta denominada Reporte Integrado (RI) o Integrated Reporting (IR, por sus siglas en inglés) es hoy la mayor innovación sobre reporting corporativo (Rivera-Arrubla, Zorio-Grima y García-Benau, 2016) y se puede definir como una comunicación concisa acerca de cómo la estrategia, el gobierno, el desempeo y los prospectos de una organización, en el contexto de su entorno, conducen a la creación de valor a lo largo del tiempo (IIRC, 2013). Es decir, ahora con el RI se puede hablar de un reporte que permita de manera integrada conocer el desempeo global de la organización.

Estas propuestas de reportes de RSC, incluyendo el RI, han sido objeto de diferentes críticas, sobre todo por el hecho de ser voluntarias, pues al no estar reguladas por ley, las corporaciones tienen una considerable libertad para decir el contenido y la forma en que lo presentan (Sandberg y Holmlund, 2015). La información que revela la organización se puede tomar como una forma de gestionar la percepción e impresión que los diferentes stakeholders tienen de ella (Merkl-Davies, Brennan y McLeay, 2011; Schleicher, 2012).

Con el RI es posible evaluar, por tanto, si la organización y su modelo de negocio propiciaron un aumento, disminución o transformación de los recursos y las relaciones en el desarrollo de su modelo de negocio, es decir, si la organización, en el desarrollo de sus actividades, creó o disminuyó valor en el corto, mediano y largo plazo (Rivera-Arrubla, Zorio-Grima y García-Benau, 2016). EL IIRC llama a los recursos y a las relaciones capitales, y estos pueden ser: financiero, industrial, intelectual, humano, social y relacional, y natural. La siguiente figura ilustra la forma en que se concibe y opera el RI:

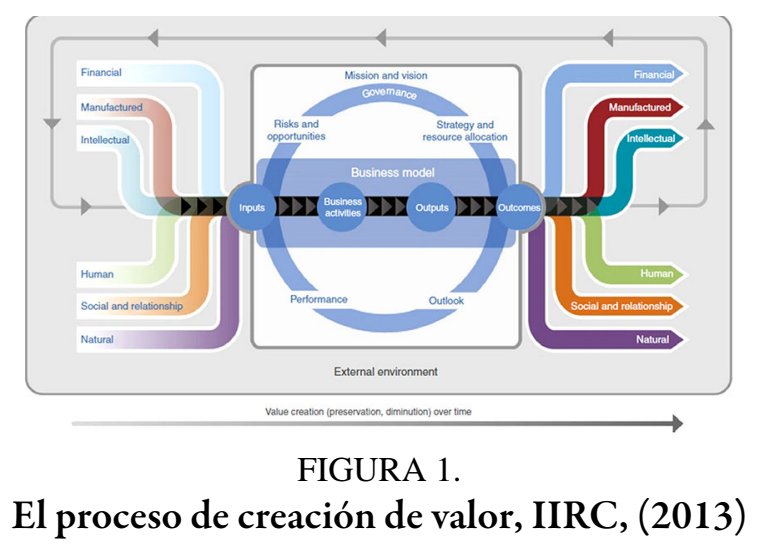

La principal razón por la que se seleccionó el RI obedece a que, como los señalan Dumay y Dai (2017), Macías y Farfán-Liévano (2017) y Rivera-Arrubla, Zorio-Grima y García-Benau (2017), además de permitir cambios sobre la forma en que las organizaciones revelan o reportan su desempeño a la sociedad, también propicia un cambio en su cultura organizacional, pues promueve una reflexión sobre cómo y en qué trabajan dichas organizaciones. Además, es la propuesta más reciente que agrega, mas no reemplaza, a las guías sobre reportes de sostenibilidad propuestas por el GRI.

En la confección de un RI participan las etapas de entradas, salidas y resultados de información financiera, de fabricación, intelectual o de conocimiento, de capital humano, de relacionales sociales e información del capital natural. Así mismo, en el RI operan las actividades de negocio que con una mirada intermedia admite 
procesar las entradas y ofrecer salidas o resultados que permiten generar el RI en las organizaciones y revelar su desempeño integral.

Hasta aquí parece un análisis descriptivo y evidente del RI, sin embargo, al asumir que en la confección del proceso de integración para su preparación están presentes de forma transversal los flujos de información y los de conocimiento, se incluye una nueva esfera de análisis, a saber: la gestión de la información y el conocimiento en la revelación de la información financiera y no financiera en un RI. Y no se trata de la gestión del conocimiento entre stakeholders después de presentar el reporte (Barkemeyer, Stringer, Hollins y Josephi, 2015), sino de evaluar el estado de dichos flujos en las organizaciones, para luego sí analizarlos en la confección del RI.

Los estudios realizados por Woodman (1985), Páez (1990), Fairer-Wessels (1997), Ponjuán (1998), Villardefrancos (2000), Noriega (2011), Alba y Herrera (2013), y el procesamiento de Alba (2015), revelan que si bien se reconoce la importancia de los flujos de información, los mismos no cuentan con una estructura definida, carecen de elementos que permitan, con una mirada integradora, aportar a los procesos organizacionales y no se integran a otras escalas superiores, como es el conocimiento o su gestión, esto contribuye a generar lo siguiente:

1. Confusión en los funcionarios por exceso de información innecesaria.

2. Divulgación e intercambio pobre, razón por la cual no circula la información.

3. Sobrecarga de información.

4. No se valora adecuadamente la información.

5. Duplicidad de información.

6. Se dificulta el acceso.

7. Bajo nivel de uso y análisis.

8. Proceso lento de toma de decisiones (Febles, 2013).

Con respecto a los flujos de conocimiento, y a pesar del interés despertado en los últimos años por los intangibles, se observa que en las organizaciones existe lo siguiente:

- La fuga de conocimiento tácito, la cual ocurre cuando una o más personas que conforman una organización salen de ella.

- Insuficientes procesos de sistematización y de recuperación de los aprendizajes.

- Poco intercambio de información no compartida, incluso entre los integrantes de la organización.

- Islas producidas por la acumulación de información y conocimiento en pocas personas, desperdiciando las buenas prácticas y las experiencias exitosas.

Para evitar estos aspectos, las organizaciones deben identificar los flujos de conocimiento y, más allá, gestionarlos, lo cual permite cumplir con uno de los desafíos que hoy enfrentan las entidades: el intercambio de conocimiento, el cual es poco logrado por la no integración correcta de las áreas que conforman las organizaciones, ocasionado, fundamentalmente, por no contar con un sistema de información capaz de compartir en tiempo real información exacta y relevante, encargado de soportar el análisis estratégico, la planeación de los escenarios y de ayudar a la gerencia a evaluar los centros de distribución, los proveedores y los servicios tercerizados (Ponjuán, 2004).

Sin embargo, en los estudios identificados se observa que las empresas no cuentan con una determinación de conocimiento, diagramas o mapas de conocimiento que permitan identificar quién en la organización puede aportar a cada tarea (Alba, 2015). Este aspecto se traslada al RI, afectando las estrategias, la asignación de recursos y el desarrollo en el general de la organización.

Bajo este contexto, en el que se busca que los flujos de información y conocimiento permitan la construcción del RI y aseguren resultados favorables en las organizaciones, se debe considerar el reconocimiento de la diversidad de competencias (saber, saber hacer, saber ser) y del desarrollo alcanzado 
en ellas por los integrantes de una organización (según Herrera et al., 2009: operativas, de desarrollo y estratégicas), generar acciones de socialización de conocimiento, capacitar a las personas de la organización para impulsar aspectos de fabricación y con ello la obtención de valor agregado a los productos y servicios que se ofrecen. Estos elementos se traducen directamente en mayores ingresos financieros y en relaciones sociales.

\section{Fundamentos teóricos de la relación modelo de Giccs: propuesta de soporte al reporte integrado}

Como secuencia de los estudios teóricos realizados a las propuestas de modelos de gestión de la información de los autores Marland (1981), Stanat (1992) citado en Villardefrancos (2005), Doyle (1992), Choo (1995), Cornella (2002), Ortoll (2003), Ponjuán (2000), (2004), Villardefrancos (2005), Artíles (2008), Carrillo (2009), Gil-Montelongo, López-Orozco y colaboradores (2011), y de gestión del conocimiento aportadas por Kogut y Zander (1992), Wiig (1993), Kim (1993), Hedlund (1994), Nonaka y Takeuchi (1995), Gopal y Gagnon (1995), Muñoz y Riverola (1997), Grant (1996), Sveiby (1997), Tejedor y Aguirre (1998), Arthur (1999), Kerschberg (2000), Pérez (2004), Soto (2005), Dájer (2006), Machado (2008), Franch y Herrera (2011), y Alba y Herrera (2013), unido a la dinámica que muestran las organizaciones y en función de establecer una propuesta concreta que permita articular las flujos de información y de conocimiento, se crea el modelo de gestión del infoconocimiento para cadenas de suministro de ron a granel (Giccs) (Alba y Herrera, 2015).

El modelo Giccs opera bajo la influencia de la gestión de la información y el conocimiento, a partir de articular las variables de mayor impacto que aparecen en los conceptos, modelos y procesos de estos enfoques. Este modelo ofrece la posibilidad de integración a los procesos de cualquier tipo de organización. El diseño se soporta en la aplicación de las tecnologías de la información y las comunicaciones, con un enfoque tecnológico que se expresa en la concepción de un portal corporativo.

Esta concepción constituye una propuesta concreta de integración, porque articula la gestión de los procesos que se llevan a cabo en una empresa bajo el criterio del infoconocimiento, el cual se encarga de integrar los flujos de información y conocimiento entre los actores, sus experiencias, el aprendizaje y las competencias, a partir del desarrollo de un pensamiento en espiral, como enfoque de proceso, creciente y sinérgico que agrega valor y crea las bases para la innovación organizacional y de productos.

El modelo identifica los elementos que desde los flujos de información y de conocimiento han de ser gestionados, generando una espiral (infoconocimiento), la cual permite la determinación y organización de la información relevante mediante el filtrado, la disponibilidad de los contenidos, el almacenamiento de fuentes y la incorporación de juicios de valor en puntos clave de la empresa, aportados por la participación de expertos y personal involucrado, los que a su vez intervienen de forma creadora en la adquisición, la comunicación y la toma de decisiones.

Las aplicaciones, la socialización y el registro de las decisiones tomadas alimentan la espiral, permitiendo generar valor a las actividades y procesos, así como promover la innovación o mejora continua, las cuales se obtienen como resultado de los continuos ciclos de la espiral.

Los resultados de estos procesos se socializan como vías para promover la colaboración, apoyados en plataformas y mecanismos informáticos que permiten acceder a los resultados y colaborar para generar transformaciones con valor agregado, mediante la transferencia, cuyos resultados se ubican en repositorios que permiten su localización y retroalimentación, aspectos que son monitoreados y administrados.

Para el caso del RI, la incorporación del Giccs en las organizaciones garantiza el impacto en tres áreas fundamentales: la gestión, el capital humano y las operaciones clave de la empresa. Los principales impactos en estas áreas están asociados con la gestión, la planificación global y la posibilidad de desagregación a los diversos eslabones que la conforman. Así mismo, está la opción de la toma de decisiones oportunas, al brindar 
de forma actualizada y sistemática la información necesaria y precisa que refuerza la articulación entre cada área y procesos de la organización. Asociado con la toma de decisiones y los recursos se promueve el desarrollo de la colaboración con un espíritu anticipador, lo cual pretende ofrecer soluciones a posibles problemas para minimizar su magnitud.

Otra área en la que se obtienen resultados es en el desarrollo del capital humano, toda vez que el modelo y su soporte informático inciden en el desarrollo de capacidades, el aprendizaje, la comunicación, la colaboración y la socialización permanente, permitiendo la detección y reconocimiento de expertos, la capacitación y entrenamiento de directivos, especialistas y trabajadores, y el desarrollo de competencias necesarias, así como la creación y fomento de espacios de conocimientos e intercambios de experiencias.

Vinculado con las operaciones se asegura la mayor conectividad entre actores y procesos, logrando la simultaneidad de las tareas y la reducción de desconexiones entre procesos y actividades, ya que su diseño asume el planteamiento de flujos de operaciones en los cuales, desde diferentes escenarios, se podrá digitar (captar), procesar y mostrar información, ejecutar acciones y obtener retroalimentación de las decisiones tomadas. Lo anterior constituye un mecanismo de control de la cadena y del propio soporte, pues al estandarizar las entradas y registros de información, su conservación y circulación, contribuye a la confiabilidad, disminuyendo los errores de los usuarios y los fraudes.

Como parte de las operaciones, el modelo integra la información y el conocimiento en un solo ciclo que permite alertar, a partir del monitoreo, la experiencia, los debates de situaciones previsibles o de posibles acciones a realizarse. Está soportado por un bloque de notificaciones que se encarga de vigilar y dar a conocer los principales indicadores de la organización, sus procesos y actividades clave, a los usuarios conectados. De esta forma, el Giccs trabaja por una gestión en tiempo real, en la que pone a disposición de sus integrantes el saber y el saber hacer de sus miembros de forma permanente (Alba y Herrera, 2015).

De ahí que el modelo Giccs para gestionar el RI constituye una propuesta concreta de articulación e integración que garantiza no solo la confección, sino que actúa de forma preventiva, asegurando que las entradas estén consolidadas, para que el RI sea favorable. Para elaborar un RI es necesario soportarse en el marco conceptual definido por el IIRC en el año 2013; este marco no presenta una orientación detallada y específica según el tipo y actividad de la empresa que lo está usando, sino que en él se establecen los principios y contenidos que rigen el contenido global de un informe integrado, así mismo explica los conceptos fundamentales que los sustentan.

Las organizaciones, en el proceso de elaboración del RI, además del marco conceptual, se pueden apoyar en las orientaciones dadas en anteriores estándares como el GRI, en las experiencias propias de reporting corporativo y, claro, en la experticia de los consultores, siempre que estén en consonancia con los lineamientos del IIRC. El marco conceptual asume un enfoque basado en principios, para reconocer las condiciones diversas de las organizaciones, así que deja en libertad a las organizaciones para organizar su RI, sin embargo, define unos requerimientos que deben ser aplicados para que el informe integrado esté de conformidad con este marco. Cualquier RI debe dar cuenta de la forma cómo una organización crea valor a lo largo del tiempo; para ello, debe informar sobre lo siguiente: los capitales y su interacción con el medio externo, y sobre las interacciones y las relaciones mediante las que la organización crea valor para los grupos de interés y la sociedad en general.

El reto mayor para los preparadores y presentadores del RI, al estar amparados por un marco basado en principios, está en definir lo siguiente: 1. qué asuntos son materiales, y 2. cómo son divulgados, incluyendo la aplicación de métodos de medición y divulgación de general aceptación, según corresponda. Como soporte, el RI cuenta con unas directrices que guían su preparación y presentación, explicando el contenido del informe y cómo se presenta la información:

- Enfoque estratégico y orientación futura.

- Conectividad de la información.

- Relación con los grupos de interés. 
- Materialidad.

- Concisión.

- Fiabilidad y exhaustividad.

- Consistencia y comparabilidad (IIRC, 2013).

La forma en que el IIRC ha propuesto la elaboración del RI es por medio de preguntas, con las cuales se asegura un nivel de información adecuado y suficiente para permitir la comparación entre organizaciones. Pero sobre todo, las preguntas están relacionadas con los contenidos del RI, que son los siguientes: descripción general de la organización y de su entorno externo, gobierno corporativo, modelo de negocio, riesgos y oportunidades, estrategia y asignación de recursos, desempeño, perspectivas, bases para la elaboración y presentación, y al hacerlo, tener en cuenta la orientación general del informe.

Para determinar qué información se reporta y cómo se reporta se deben aplicar las directrices enunciadas y las preguntas sobre los contenidos, sin embargo, es necesario resaltar que son los flujos de información y de conocimiento los que permiten la construcción del RI, es decir, cada dato, producido y analizado, cada información financiera y no financiera generada en la organización, y que por medio de diferentes procesos es analizada y revelada, está mediada por flujos de información y conocimiento.

El marco conceptual del RI tiene dentro de sus directrices un enfoque estratégico y orientación futura, y también la conectividad de la información, pero, ¿̇son suficientes para resolver los problemas enunciados sobre los modelos de gestión de la información y del conocimiento? Cuando el IIRC define la conectividad, afirma que un RI debe revelar "una imagen holística de la combinación, interrelación y dependencia entre los factores que afectan a la capacidad de la organización para crear valor a lo largo del tiempo", (2013, p. 7); con esto se entiende que el RI debe ser más que un resumen de información dentro de otra forma de comunicación y, por lo tanto, hace referencia a la integración de los flujos de información y, de forma más precisa, a la coherencia y a la relevancia de la misma. Sin embargo, no se encuentra en este marco conceptual una alusión explícita al intercambio de conocimiento y a la forma en que los flujos de información se gestionan de forma conjunta con los flujos de conocimiento.

Otro supuesto del RI que puede ser cuestionado desde el modelo Giccs es la incorporación del pensamiento integrado en las actividades de una organización; como ya se enunció, las organizaciones presentan de forma general problemas con la gestión de sus flujos de información y conocimiento, y de forma específica, con la integración en los sistemas de información, que en este caso son el soporte de los informes y la comunicación interna y externa, incluyendo la preparación del RI.

Finalmente, la pertinencia de pensar el RI desde el modelo Giccs se puede ver en relación con los flujos de conocimiento; en el RI, la alusión más cercana es la mención que se hace al capital intelectual, pero, ¿cómo pensar el capital intelectual con una mirada de flujos de conocimiento? A los capitales los define el IIRC como "inventarios de valor que se incrementan, disminuyen y transforman a través de las actividades y las salidas de la organización" (2013, p. 6), por tanto, esta definición da una mirada limitada que los restringe a la información sobre un tipo de capital (intangibles basados en el conocimiento de la organización), más no como la gestión de un flujo vital en las organizaciones.

\section{Aproximaciones prácticas: el reporte integrado en el modelo de Giccs}

Para asumir el Giccs dentro de la concepción de un RI se revisaron sus conceptos fundamentales, directrices y contenidos, demostrando que existe total compatibilidad con el modelo Giccs; la información, que es el insumo fundamental del RI, también hace parte de Giccs; la diferencia del RI con el Giccs radica entonces en la gestión del conocimiento, pero, sobre todo, en la gestión conjunta que se hace. El RI, con claridad, identifica la información que será incluida para evaluar la habilidad de la organización para crear valor, sin embargo, cuando involucra los elementos propios del conocimiento, hace referencia explícita a él como un 
capital con naturaleza intangible, y en relación con la gestión del conocimiento en el proceso de preparación y presentación, solo afirma que este debe ser de manera colectiva y que debe estar dentro de la declaración de los responsables de gobierno corporativo.

El RI es un aliado fundamental en la identificación de los flujos de información y conocimiento que deben ser gestionados, por lo que constituye un insumo en la puesta en marcha del modelo Giccs. Sin embargo, su incorporación en las organizaciones requiere asumir un conjunto de principios:

- Sistémico: significa que la integración de la GI con la GC es más que la suma simple de sus partes o procesos clave; es una nueva cualidad surgida de la interacción de sus partes, reconocida como infoconocimiento, con un ciclo y una dinámica propia.

- Interdisciplinario: demanda la acción de diferentes disciplinas científicas, errando quien pretenda sesgarla con el predominio de alguna. La concepción relaciona las disciplinas de gestión por proceso, competencias, sistemas de información, talento humano, conocimiento y tecnología de información.

- Transdisciplinario: expresa la transversalidad de su accionar en los distintos procesos de la cadena de suministro.

- Colaborativo: articula las informaciones que se generan desde los diversos actores y escenarios y ofrece reportes con alto valor de conocimiento que permiten mejorar las bases para la toma de decisiones.

- Proactividad: señala la actuación anticipada, contraria a la reactiva, lo cual es promovido por el enfoque de GC con el que es gestionado y el soporte para el registro y la inmediatez, por lo que se crean las condiciones para reducir las respuestas cuando se presenta el problema o, peor aún, después de su manifestación. El registro y monitoreo permiten alertas tempranas para la toma de decisiones.

- Procesos: sigue la secuencia de actividades de la CS, en donde se analiza el encadenamiento del flujo de información y conocimiento.

- Competencias laborales: considera la gestión del CH a partir de las habilidades, destrezas y saberes que portan las personas que trabajan en la cadena de suministro.

- Tecnológico: las operaciones de la cadena se gestionan desde un soporte tecnológico que asimila avances significativos en materia de conocimiento e interactividad.

Los principios que rigen el diseño y funcionamiento del Giccs no son suficientes para su aplicabilidad, se requiere de la existencia de un conjunto de premisas que hagan posible los insumos y puesta en práctica de este modelo; tales premisas se definen como:

1. Identificación de la cadena de suministro: reconocimiento de la dependencia funcional y sistemática entre las entidades para el éxito de un negocio.

2. Existencia de manuales o registros de procedimientos: disponer de memoria gerencial y de proceso que documenten las operaciones de la cadena y de sus actores.

3. Disponer de los recursos tecnológicos para la puesta en marcha del portal: contar o prever la adquisición de medios informáticos para la introducción del portal.

En función de estas premisas se determinan como parte de esta investigación las compatibilidades con el RI:

1. Identificación de la cadena de suministro: tanto en los contenidos que deben ser informados, como en su esencia, el RI hace alusión a la cadena de suministro, pues considera que la capacidad de una organización para crear valor para sí misma está relacionada con el valor que genera para los demás (IIRC, 2013).

2. Existencia de manuales o registros de procedimientos: el RI se basa en la información y los sistemas que tiene las organizaciones para gestionarla, por esta razón se asume que existen procesos que documenten las actividades de la organización y de la cadena en la que se encuentra inmersa. Esta 
idea se refuerza con la declaración de responsabilidad para garantizar la integridad del informe que deben hacer los miembros del gobierno corporativo de la organización que está divulgando su RI.

3. Recursos tecnológicos para la puesta en marcha del portal: el uso de internet para divulgar información voluntaria se ha incrementado de forma exponencial en los últimos años, permitiendo ahora a los inversionistas individuales acceder a información antes reservada solo para inversionistas institucionales y analistas (García, Sánchez e Isabel, 2011). El RI no solo pone a disposición su base de datos para hacer visibles los RI de las organizaciones y sugiere el uso de portales institucionales para darlos a conocer, sino que además está comprometido con la inclusión de la tecnología y por esto ha propuesto la Technology Initiative (TI), que busca involucrar las nuevas tendencias en el reporte corporativo y, en particular, pueden aplicarse para ayudar en la adopción global de informes integrados (IIRC, 2016).

En función de los principios y premisas identificados para el Giccs y con el objetivo de declarar los puntos de contacto entre este modelo y el RI, se han definido las funcionalidades que garantiza el primero, así como los puntos en los que asegura la entrada, procesamiento o salida del segundo.

El Giccs logra gestionar en las organizaciones los siguientes procesos:

1. Tramitar información sobre presupuesto, pedidos, preparación, fabricación, producción, almacenamiento, despacho, experiencias, aprendizaje entre expertos y competencias del capital humano.

2. Monitorear y establecer la trazabilidad de la información en tiempo real.

3. Generar reportes a partir de la minería de datos.

Estos elementos antes identificados permiten conocer que el Giccs cumple con las necesidades del RI y, por tanto, su inclusión brinda beneficios como los siguientes:

- El RI tendría unos flujos de información integrados y basados en el conocimiento para los informes de gestión, análisis y toma de decisiones, pues el Giccs permite la determinación y organización de la información relevante mediante el filtrado, la disponibilidad de los contenidos, el almacenamiento de fuentes y la incorporación de juicios de valor en puntos clave de la empresa.

- Mejorar la integración en los sistemas de información que soportan los informes, pues el modelo integra la información y el conocimiento en un solo ciclo que permite alertar, a partir del monitoreo, la experiencia y los debates de situaciones previsibles o de posibles acciones a realizarse.

Por último, impulsa el cumplimiento del último objetivo del RI, pues con la inclusión del modelo Giccs no solo se alcanzan cambios en la forma en que las organizaciones revelan o reportan su desempeño a la sociedad, sino que también se favorece un cambio en su cultura organizacional, ya que con este modelo se genera una mayor conectividad entre actores y procesos, logrando la simultaneidad de las tareas y la reducción de desconexiones entre procesos y actividades.

\section{Conclusiones}

1. El marco conceptual es un marco general y flexible para cualquier tipo de organización privada, por tal razón no establece indicadores clave de desempeño específicos, ni métodos de medición, ni la divulgación de asuntos particulares y tampoco pretende generar clasificaciones o parámetros mínimos para calificar a las organizaciones. Sin embargo, como resultado de esta investigación se sugiere que el marco conceptual debería incluir en su concepción unas orientaciones precisas para gestionar los flujos de conocimiento, como lo propone el modelo Giccs, pues es necesario que el 
RI, con este nuevo enfoque, trascienda la concepción del conocimiento como un capital referido de manera precisa a los intangibles, a una concepción de gestión del infoconocimiento.

2. Es necesario gestionar los flujos de información y conocimiento de forma conjunta, como lo plantea el modelo Giccs; las organizaciones presentan de forma general problemas con la gestión de sus flujos de información y conocimiento, y de forma específica con la integración en los sistemas de información, que en este caso son el soporte de la información para producir los informes y la comunicación interna y externa, incluyendo el RI.

3. EL RI es un aliado fundamental en la identificación de los flujos de información y conocimiento que deben ser gestionados, de tal suerte que por medio del modelo Giccs se pueda definir una estructura clara para estos flujos, integrar elementos para aportar a los procesos organizacionales y finalmente permitir su integración a escalas superiores.

4. El reporte integrado requiere una gestión de los flujos de información y de conocimiento de las áreas y procesos de la organización, sin embargo, la dinámica actual carece de elementos, como los mapas de conocimiento y los flujos de conocimiento, que permitan confeccionar y obtener resultados favorables para el reporte integrado.

5. El modelo de Giccs constituye una propuesta concreta de integración de los flujos de información y de conocimiento, porque logra articular estos flujos con las experiencias, el saber hacer, los procesos organizacionales y el aprendizaje.

\section{Agradecimientos}

Financiado por: Universidad Externado de Colombia, Grupo SICO.

\section{Referencias bibliográficas}

Alba, M. y Herrera, K. C. (2015). Modelo de gestión del infoconocimiento para cadenas de suministro de ron a granel. La Habana: tesis en opción al grado científico de doctora en ciencias económicas. Centro de Estudios de Técnicas de Dirección. Universidad de La Habana.

Alba, M. y Herrera, K. C. (2013). Diseño de un portal corporativo para la gestión del conocimiento en la cadena del ron granel Havana Club. La Habana: tesis en opción del título de máster en Gestión de información. Facultad de Economía. Universidad de La Habana.

Arthur, A. (1999). El management en el siglo XXI. Herramientas para los desafios empresariales de la próxima década. Buenos Aires: Granica.

Artíles, S. (2008). Modelo de gestión integrada de información y conocimiento para la empresa cubana en perfeccionamiento. La Habana: tesis para optar el grado científico de doctor en ciencias.

Barkemeyer, R., Stringer, L. C., Hollins, J. A. y Josephi, F. (2015). Corporate Reporting on Solutions to Wicked Problems: Sustainable Land Management in the Mining Sector. Environmental Science and Policy, 48, 196-209. 10.1016/j.envsci.2014.12.021 Recuperado de https://www.sciencedirect.com/science/article/pii/S146290111 4002494

Bruhn, M. y Zimmermann, A. (2017). Integrated CSR Communications. En S. Diehl, B. Mueller, F. Weder, M. Karmasin y R. Terlutter, Handbook of Integrated CSR Communication (pp. 3-21). Switzerland: Springer International Publishing.

Carrillo, R. (2009). Modelo para perfeccionar la gestión de información en la Escuela Militar Superior "Comandante Arides Estévez Sánchez". La Habana: tesis presentada en opción al título de máster en ciencias de la información y bibliotecología.

Choo, C. (1995). Information Management for the Intelligent Organization: Roles and Implications for the Information Professions. Singapore: Digital Libraries Conference. 
Cornella, A. (2002). La gestión inteligente de la información en las organizaciones. España: Ediciones.

Dájer, J. (2006). Modelo para la gestión del conocimiento en los destacamentos fronterizos del país. Tesis para optar por el título de doctor en ciencias técnicas, Facultad de Ingeniería Industrial-Economía, Ingeniería Industrial, Matanzas, Cuba.

Doyle, C. (1992). Outcome Measures for Information Literacy within the National Education Goals of 1990. Final Report to National Forum on Information Literacy. Summary of Indings.

Dumay, J. y Dai, T. (2017). Integrated Thinking as a Cultural Control? Meditari Accountancy Research, 25 (4), 574-604. 10.1108/MEDAR-07-2016-0067. Recuperado de: https://www.scopus.com/inward/record.uri?eid=2-s2.0-85031111767\&doi=10.1108\%2fMEDAR-07-20 16-0067\&partnerID=40\&md5=0035161e3f258ebdec41179db0a98ecb

Fairer-Wessels, F. A. (1997). Information Management Education: Towards a Holistic Perspective. South African Journal of Library and Information Science, 93-102.

Febles, J. (2013). La gestión del conocimiento y herramientas inteligentes. La Habana: inédito. Presentación en la maestría de Gestión de información.

Franch, K. y Herrera, K. C. (2011). La gestión del conocimiento como herramienta de apoyo al proceso de toma de decisiones: caso de estudio Dirección General TRD Caribe. Universidad de La Habana. La Habana: Ceted.

García, I., Sánchez, L. e Isabel, G. (2011). Corporate Governance and Strategic Information on the Internet: A Study of Spanish Listed Companies. Accounting, Auditing \& Accountability Journal, 24, 471-501.

Gil-Montelongo, M. D., López-Orozco, G. y colaboradores. (2011). Proceso de gestión de información (información con base al conocimiento).

Gopal y Gagnon. (1995). Knowledge, Information, Learning and the IS Manager. Computerword (leadership series), $1(5), 1-7$.

Grant, R. (1996). Prospering in Dynamically-Competitive Environments: Organizational Capability as Knowledge Integration. Organization Science, 7 (4) (julio-agosto), 375-387.

Hahn, R. y Kuhnen, M. (2013). Determinants of Sustainability Reporting: a Review of Results, Trends, Theory, and Opportunities in an Expanding Field of Research. Journal of Cleaner Production, 59, 5-21. 10.1016/ j.jclepro.2013.07.005

Hedlund, G. (1994). A Model of Knowledge Management and N-Form Corporation. Strategic Management Journal, 15, 73-90.

Herrera, K. C., Rodríguez, F., Franch, K. y Montejo, R. (2009). Introducción de la NC 3000 en la empresa productora de alimentos de Regla-Prodal. Universidad de La Habana, Centro de Estudios de Técnicas de Dirección. La Habana: Ceted.

IIRC. (2013). The International Framework. London: International Integrated Reporting Council (IIRC).

IIRC. (2016). Technology Initiative. London: International Integrated Reporting Council.

Ijiri, Y. (1976). Análisis de objetivos y control de gestión: criterios para los procesos de planificación y control de gestión. Madrid: ICE.

Kerschberg, L. (2000). Knowledge Management: Managing Knowledge Resources for the Intelligent Enterprise. Chile: XXIII Taller de Ingeniería de Sistemas.

Kim, D. H. (1993). The Link Between Individual and Organizational Learning. Sloan Management Review. Fall, 37-50.

Kogut y Zander. (1992). Knowledge of de Firm, Combinative Capabilities and the Replication of the Technology. Organization Science, 3 (3), 383-397.

KPMG. (2017). The KPMG Survey of Corporate Responsibility Reporting 2017. Recuperado de: https://home.kpmg. com/content/dam/kpmg/campaigns/csr/pdf/CSR_Reporting_2017.pdf

Machado, R. (2008). Quépodemos entender por capital humano, cómo crearlo y gestionarlo en las condiciones de Cuba. La Habana: Ediciones Balcón Cidtur Eaeht. 
Macías, H. A. y Farfán-Liévano, A. (2017). Integrated Reporting as a Strategy for Firm Growth: Multiple Case Study in Colombia. Meditari Accountancy Research, 25 (4), 605-628. 10.1108/MEDAR-11-2016-0099 Recuperado de https://www.scopus.com/inward/record.uri?eid=2-s2.0-85031099765\&doi=10.1108\%2fMEDAR-11-201 6-0099\&partnerID=40\&md5=a4f72f64a936a2614396722aa9358ff8

Marland, M. (1981). Information Skills in the Secondary Curriculum. London: Methuen.

Merkl-Davies, D. M., Brennan, N. M. y McLeay, S. J. (2011). Impression Management and Retrospective Sense-Making in Corporate Narratives. Accounting, Auditing \& Accountability Journal, 24 (3), 315-344. 10.1108/09513571111124036. Recuperado de: http://www.emeraldinsight.com/doi/abs/10.1108/09513571 111124036

Muñoz Seca, B. Y. (1997). Gestión del conocimiento. Barcelona, España: Universidad de Navarra.

Nonaka y Takeuchi. (1995). The Knowledge-Creating Company: How Japanese Companies Create the Dynamics for Innovation. Nueva York: Oxford University Press.

Noriega, Y. (2011). Sistema de gestión de información automatizado para instalaciones patrimoniales del Minint. La Habana: trabajo de diploma para optar por el título de ingeniero en informática. Instituto Superior Politécnico José A. Echevarría.

Ortoll, E. (2003). Gestión del conocimiento y competencias informacionales en el puesto de trabajo.

Páez, I. (1990). Información para el progreso de América Latina. Caracas: Coediciones Universidad Simón Bolívar.

Pérez, A. (2004). Sobre el nuevo sistema de dirección empresarial. Nueva empresa, Revista Cubana de Gestión Empresarial, 0 (1), 5-9.

Ponjuán, G. (2004). Gestión de información: dimensiones e implementación para el éxito organizacional. 1 ed. Rosario, Argentina: Nuevo paradigma.

Ponjuán, G. (2000). Aplicaciones de gestión de información en las organizaciones. El profesional de la información y su dominio de las técnicas y herramientas de gestión. La Habana: tesis en opción del grado de doctor en ciencias de la información.

Ponjuán, G. (1998). Gestión de información en las organizaciones. Conceptos, principios y aplicaciones. Santiago de Chile: Cecapi.

Rivera-Arrubla, A., Zorio-Grima, A. y García-Benau, M. A. (2016). El concepto de informe integrado como innovación en reporting corporativo. Journal of Innovation \& Knowledge, 144-155.

Sandberg, M. y Holmlund, M. (2015). Impression Management Tactics in Sustainability Reporting. Social Responsibility Journal, 677-689.

Sierra-García, L., García-Benau, M. A. y Zorio, A. (2014). Credibilidad en Latinoamérica del informe de responsabilidad social corporativa. Innovar, 28-38.

Schleicher, T. (2012). When is Good News Really Good News? Accounting and Business Research, 42 (5), 547-573. 10.1080/00014788.2012.685275. Recuperado de http://www.tandfonline.com/doi/abs/10.1080/00014788. 2012.685275

Soto, M. A. (2005). Modelación de la gestión del conocimiento para las organizaciones cubanas a través de los portales de información. Tesis para optar por el grado científico de doctor en ciencias de la información, La Habana, Cuba.

Sunder, S. (2005). Teoría de la contabilidad y el control. Bogotá: Universidad Nacional de Colombia.

Sveiby, K. E. (1997). The New Organizational Wealth. Estados Unidos: Berett-Koehler.

Tejedor, B. y Aguirre, A. (1998). Proyectos logos, investigación relativa a la capacidad de aprender de las empresas españolas. Boletín de estudios económicos, LIII (164), 231-249.

Toppinen, A., Li, N., Tuppura, A. y Xiong, Y. (2012). Corporate Responsibility and Strategic Groups in the ForestBased Industry: Exploratory Analysis Based on the Global Reporting Initiative (GRI) Framework. Corporate Social Responsibility and Environmental Management, 19 (4), 191-205. 10.1002/csr.256

Villardefrancos, M. D. (2000). Estudio del flujo de información para la optimización de procesos en la Facultad de Comunicación. La Habana: tesis para optar por el título de máster en gestión de información en las organizaciones. 
Wiig, K. (1993). Knowledge Management Foundations: Thinking About Thinking-How People and Organizations Create, Represent and Use of Knowledge. Arlington: Schema Press.

Woodman, L. (1985). Information Management in Large Organizations. London: Cronin, B., ed. Information Management: From Strategies to Action. Aslib.

Licencia Creative Commons CC BY 4.0

Para citar este artículo: Alba, M., y Beltrán-Torres, C. (2018). Articulación de procesos, flujos de información y conocimiento bajo criterios de infoconocimiento y sostenibilidad en el reporte corporativo. Cuadernos de Contabilidad, 19(47), 117-129. https://doi.org/10.11144/Javeriana.cc19-47.apfi 